10-20-1997

\title{
Hybrid Reflection-Transmission Surface Light-Scattering Instrument with Reduced Sensitivity to Surface Sloshing
}

\author{
William V. Meyer
}

James A. Lock

Cleveland State University, j.lock@csuohio.edu

H. Michael Cheung

Follow this and additional works at: https://engagedscholarship.csuohio.edu/sciphysics_facpub

Tromas W. Taylor

CRartof shate Phiversity, t.tayllor@csuohio.edu

How does access to this work benefit you? Let us know!

Padethisheir's Statement

This paper was published in Applied Optics and is made available as an electronic reprint with

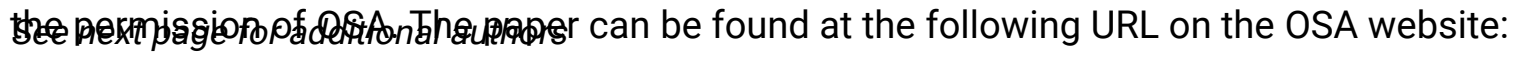
http://www.opticsinfobase.org/ao/abstract.cfm?URI=ao-36-30-7605. Systematic or multiple reproduction or distribution to multiple locations via electronic or other means is prohibited and is subject to penalties under law.

\section{Original Citation}

Meyer, William V., James A. Lock, H. Michael Cheung, Thomas W. Taylor, Padetha Tin, and J. Adin Mann. "Hybrid Reflection-Transmission Surface Light-Scattering Instrument with Reduced Sensitivity to Surface Sloshing." Applied Optics 36 (1997): 7605-7614.

\section{Repository Citation}

Meyer, William V.; Lock, James A.; Cheung, H. Michael; Taylor, Thomas W.; Tin, Padetha; and Mann, J. Adin, "Hybrid Reflection-Transmission Surface Light-Scattering Instrument with Reduced Sensitivity to Surface Sloshing" (1997). Physics Faculty Publications. 106.

https://engagedscholarship.csuohio.edu/sciphysics_facpub/106

This Article is brought to you for free and open access by the Physics Department at EngagedScholarship@CSU. It has been accepted for inclusion in Physics Faculty Publications by an authorized administrator of EngagedScholarship@CSU. For more information, please contact library.es@csuohio.edu. 
Authors

William V. Meyer, James A. Lock, H. Michael Cheung, Thomas W. Taylor, Padetha Tin, and J. Adin Mann 


\title{
Hybrid reflection-transmission surface light-scattering instrument with reduced sensitivity to surface sloshing
}

\author{
William V. Meyer, James A. Lock, H. Michael Cheung, Thomas W. Taylor, Padetha Tin, \\ and J. Adin Mann, Jr.
}

\begin{abstract}
A hybrid reflection-transmission surface light-scattering instrumental design is presented, examined theoretically, and tested experimentally. The purpose of the design is to reduce the sensitivity of the instrument to vibration in general and surface sloshing in particular while sacrificing little performance. Traditional optical arrangements and two new optical configurations with varying trade-offs between slosh resistance and instrumental simplicity and accuracy are examined by use of Fourier optics methods. The most promising design was constructed and tested with acetone, ethanol, and water as subject fluids. The test involved backcalculation of the wave number of the capillary wave examined with the known physical parameters for the test fluids. The agreement of the computed wave number was $\pm 1.4 \%$. (C) 1997 Optical Society of America
\end{abstract}

\section{Introduction}

When a liquid of refractive index $n$ in a large open dish is isolated from all external vibrations, the surface of the liquid appears static to the unaided eye. But random thermal motions of molecules near the liquid surface continually provide small-amplitude pressure fluctuations that generate capillary waves that then propagate along the liquid surface and gradually dissipate. ${ }^{1}$ Surface capillary waves may also be generated either mechanically or by the application of oscillating electric-field gradients across the liquid surface. In each case the behavior of the capillary waves is described by the same fluid mechanical theory. The statistical properties of surface capillary waves can be nonintrusively measured by the scattering of laser light from the liquid surface. The frequency of the scattered light is Doppler shifted slightly because of the capillary-wave motion. When the scattered electric field is combined at a

W. V. Meyer and P. Tin are with the Ohio Aerospace Institute, NASA Lewis Research Center, Cleveland, Ohio 44135. J. A. Lock and T. W. Taylor are with the Department of Physics, Cleveland State University, Cleveland, Ohio 44115. H. M. Cheung is with the Department of Chemical Engineering, University of Akron, Akron, Ohio 44325. J. A. Mann, Jr. is with the Department of Chemical Engineering, Case Western Reserve University, Cleveland, Ohio 44106.

Received 13 March 1997; revised manuscript received 22 May 1997.

0003-6935/97/307605-10\$10.00/0

(C) 1997 Optical Society of America detector with a reference beam that has the original laser frequency, the time variation of the recorded intensity may be processed by means of autocorrelation function techniques to obtain information about the response of the liquid surface to small fluctuations.

Previous surface light-scattering experiments have employed a large variety of scattering geometries and types of instrumentation. ${ }^{2-12}$ But they have all shared a common concern, namely, when the liquid surface as a whole sloshes in response to external low-frequency vibrations caused by passing traffic or people walking in the laboratory, the scattered light beam deflects back and forth past the detector face. As a result, careful precautions are taken to ensure that the open dish containing the liquid is isolated to a high degree from external vibrations. Progress has also been made in designing surface lightscattering systems that are tolerant to sloshing. In traditional reflection experiments, when the surface of the liquid tilts by an angle $\phi$, the reflected beam deflects by $2 \phi$ and may easily leave the detector face. But in transmission experiments, ${ }^{10}$ the beam deflects by only $(n-1) \phi$, which for water is only $1 / 6$ of the deflection encountered in reflection systems.

In this paper we both theoretically analyze and experimentally test a new surface light-scattering instrument that also has decreased sensitivity to surface sloshing. We employ a hybrid reflectiontransmission system in which a spatially filtered and expanded incident laser beam is transmitted through the liquid, reflected by a spherical mirror 

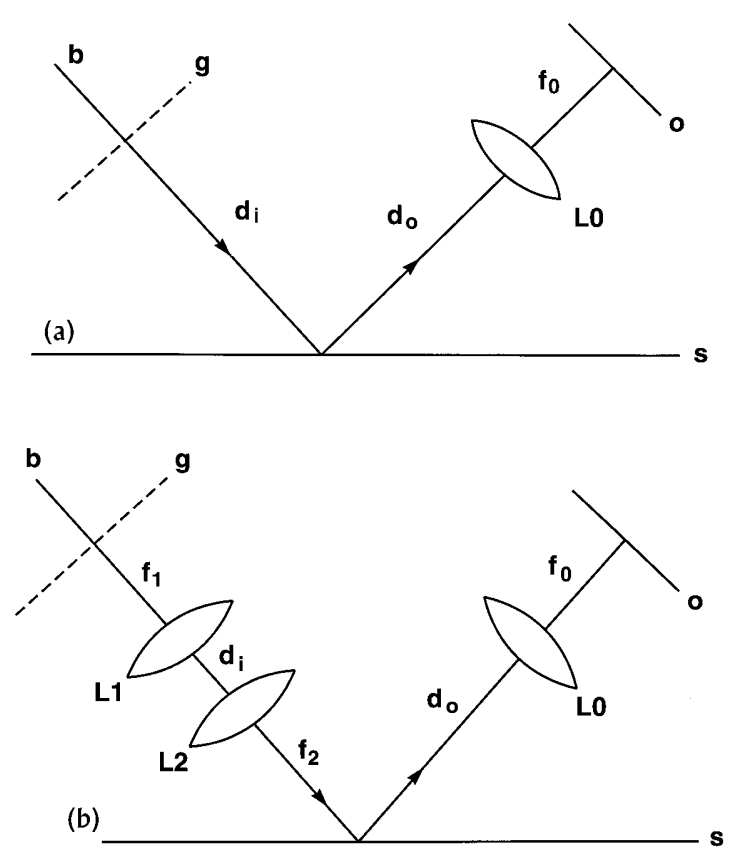

Fig. 1. (a) Gaussian laser beam $b$ is transmitted through a phase grating g, reflects off of a liquid surface $\mathrm{s}$, and is focused by collection lens $\mathrm{L} 0$ onto the detector plane 0 . The distances between the grating and the surface and between the surface and lens L 0 are $d_{i}$ and $d_{o}$, respectively. (b) The Gaussian laser beam is imaged onto the liquid surface by lenses L1 and L2.

below the transparent liquid-containing dish, and is transmitted back through the liquid a second time. This system is based on the idea of using the spherical mirror as a retroreflector and is called a cat's eye system. ${ }^{13}$ But in order to obtain an easily analyzable homodyne signal, the retroreflecting properties of the spherical mirror must be compromised somewhat. As a result, when the liquid surface tilts by an angle $\phi$ for our placement of the mirror, the final scattered beam deflects by approximately $(n-1) \phi$, which is comparable with the deflection for the single-pass transmission geometry of Ref. 10.

The body of this paper proceeds as follows. We theoretically analyze the laser beam propagation and surface scattering by using Fourier optics. ${ }^{14}$ The application of Fourier optics methods to surface light scattering was pioneered by Lading et al., ${ }^{9}$ who considered a generalization of the system shown in Fig. 1 wherein spatial filters could be inserted at the various focal planes to tailor the beam reaching the detector in several ways, including the selection of the homodyne signal or the heterodyne signal. In Section 2 we supply the details omitted in the general procedure outlined in Ref. 9 for the traditional reflection geometry. Then in Section 3 we analyze the hybrid reflection-transmission geometry. In Section 4 we describe our experimental setup and demonstrate its reduced sensitivity to surface sloshing. Finally, in Section 5 we discuss our results. In Appendix A we derive two significant corrections to the results of Section 2 that previous treatments have not considered.

\section{Fourier Optics Analysis of the Traditional Surface Light-Scattering Geometry}

In this section we consider the traditional reflection geometries of Figs. 1(a) and 1(b). In each case a monochromatic filtered and expanded Gaussian laser beam (b) is transmitted through a phase grating $(\mathrm{g})$ and approaches the liquid surface (s) with the angle of incidence $(\alpha)$. In Fig. 1(a) the light transmitted through the grating reflects from the surface. In Fig. 1(b) the grating transmissivity is imaged onto the surface by lenses L1 and L2. In each case the light reflected from the surface is collected by a largeaperture spherical lens (L0) and is focused onto the detector plane (o).

The optical properties of the beam, phase grating, liquid surface, and collection lens are as follows. A Gaussian beam propagates along the $z_{i}$ axis. Its electric field at the entrance plane of the phase grating is

$$
E\left(\mathbf{r}_{i}\right)=E_{\text {inc }} \exp \left(-r_{i}^{2} / w_{g}{ }^{2}\right) \exp \left(i Q_{g} r_{i}^{2}\right)
$$

where

$$
\mathbf{r}_{i}=x_{i} \hat{u}_{x}^{i}+y_{i} \hat{u}_{y}{ }^{i},
$$

$E_{\text {inc }}$ is the incident electric-field strength, $w_{g}$ is the electric-field 1/e half-width of the beam at the grating, and $Q_{g}$ describes the curvature of the spherical wave fronts there. The electric-field transmissivity of the phase grating is

$$
\begin{aligned}
T\left(\mathbf{r}_{i}\right)= & \exp \left(i \epsilon \cos \mathbf{K} \cdot \mathbf{r}_{i}\right) \approx 1+\frac{i \epsilon}{2} \exp \left(i \mathbf{K} \cdot \mathbf{r}_{i}\right) \\
& +\frac{i \epsilon}{2} \exp \left(-i \mathbf{K} \cdot \mathbf{r}_{i}\right),
\end{aligned}
$$

where $\epsilon$ is the grating's diffractive power $(\epsilon \approx 0.1$ is usually desired). The grating is oriented so that its rulings make an angle $\eta$ with the $x_{i}$ axis, and the grating wave vector $\mathbf{K}$ is related to the ruling spacing $l$ on the grating by

$$
\mathbf{K}=\frac{2 \pi}{l}\left(\cos \eta \hat{u}_{x}^{i}+\sin \eta \hat{u}_{y}^{i}\right) .
$$

The transmitted electric field at the grating's exit plane is $E\left(\mathbf{r}_{i}\right) T\left(\mathbf{r}_{i}\right)$ and consists of the incident Gaussian beam modulated by the three spatial frequencies of the grating. As it propagates beyond the grating toward the liquid surface, the transmitted beam rapidly evolves into a central undiffracted Gaussian beam and two-grating first-order diffracted Gaussian beams.

These beams are then incident upon a reflecting surface whose shape is $z_{s}\left(x_{s}, y_{s}\right)$. The surface $(s)$, incoming $(i)$, and outgoing $(o)$ coordinate systems in 


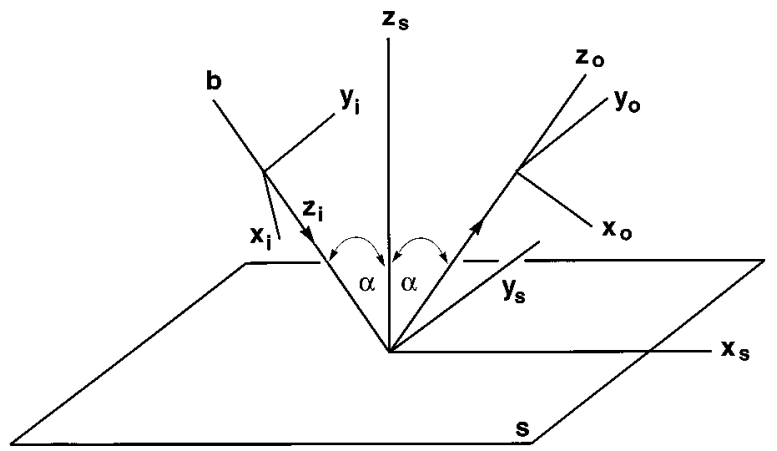

Fig. 2. Beam $b$ specularly reflects at an angle $\alpha$ from the flat surface $\mathrm{s}$. The coordinates $\left(x_{i}, y_{i}, z_{i}\right)$ describe the incoming beam, and the coordinates $\left(x_{o}, y_{o}, z_{o}\right)$ describe the outgoing beam.

Fig. 2 have a common origin and are rotated with respect to each other by means of

$$
\begin{aligned}
& x_{i}=-x_{s} \cos \alpha-z_{s} \sin \alpha, \\
& y_{i}=y_{s}, \\
& z_{i}=x_{s} \sin \alpha-z_{s} \cos \alpha ; \\
& x_{o}=x_{s} \cos \alpha-z_{s} \sin \alpha, \\
& y_{o}=y_{s}, \\
& z_{o}=x_{s} \sin \alpha+z_{s} \cos \alpha ;
\end{aligned}
$$

By examining the time delay of the light from its incidence upon the surface to its arrival at the point $x_{o}, y_{o}, z_{o}$ on the detector plane, we obtain

$$
\begin{aligned}
E\left(x_{o}, y_{o}, z_{o}, t\right)= & \frac{-i k E_{\text {inc }} \cos \alpha}{2 \pi z_{o}} \exp \left(i k z_{o}-i \omega t\right) \\
& \times \int \mathrm{d} x_{s} \mathrm{~d} y_{s} F_{\text {surface }}\left(x_{i}, y_{i}, z_{i}\right) \\
& \times \exp \left(-2 i k z_{s} \cos \alpha\right) \\
& \times \exp \left[i k \left(x_{o}-x_{s} \cos \alpha\right.\right. \\
& \left.\left.+z_{s} \sin \alpha\right)^{2} / 2 z_{o}\right] \\
& \times \exp \left[i k\left(y_{o}-y_{s}\right)^{2} / 2 z_{o}\right]
\end{aligned}
$$

where $F_{\text {surface }}$ is the incident-beam profile evaluated on the reflecting surface. If the surface everywhere has a small slope and $\alpha$ is not near grazing incidence, Eq. (7) may be simplified to

$$
\begin{aligned}
E\left(x_{o}, y_{o}, z_{o}, t\right)= & \frac{-i k E_{\text {inc }} \cos \alpha}{2 \pi z_{o}} \exp \left(i k z_{o}-i \omega t\right) \\
& \times \int \mathrm{d} x_{s} \mathrm{~d} y_{s} F_{\text {surface }}\left(x_{i}, y_{i}, z_{i}\right) \\
& \times \exp \left(-2 i k z_{s} \cos \alpha\right) \\
& \times \exp \left[i k\left(x_{o}-x_{s} \cos \alpha\right)^{2} / 2 z_{o}\right] \\
& \times \exp \left[i k\left(y_{o}-y_{s}\right)^{2} / 2 z_{o}\right] .
\end{aligned}
$$

If $\left|2 k z_{s} \cos \alpha\right| \ll 1$ (for surface light scattering from thermally induced capillary waves, for example, one usually has $\left|2 k z_{s} \cos \alpha\right| \lesssim 10^{-2}$ ), Eq. (8) becomes approximately

$$
\begin{aligned}
E\left(x_{o}, y_{o}, z_{o}, t\right)= & \frac{-i k E_{\text {inc }} \cos \alpha}{2 \pi z_{o}} \exp \left(i k z_{o}-i \omega t\right) \\
& \times \int \mathrm{d} x_{s} \mathrm{~d} y_{s} F_{\text {surface }}\left(x_{i}, y_{i}, z_{i}\right) \\
& \times\left(1-2 i k z_{s} \cos \alpha\right) \\
& \times \exp \left[i k\left(x_{o}-x_{s} \cos \alpha\right)^{2} / 2 z_{o}\right] \\
& \times \exp \left[i k\left(y_{o}-y_{s}\right)^{2} / 2 z_{o}\right] .
\end{aligned}
$$

As a specific example, we consider light scattered by a surface capillary wave of amplitude $A_{0}$, wavelength $\Lambda$, angular frequency $\Omega$, and wave vector

$$
\mathbf{p}=\frac{2 \pi}{\Lambda}\left(\cos \xi \hat{u}_{x}^{s}+\sin \xi \hat{u}_{y}^{s}\right),
$$

whose crests make an angle $\xi$ with the $x_{s}$ axis of Fig. 2. The liquid surface profile of the capillary wave is

$$
\begin{aligned}
z_{s}\left(\mathbf{r}_{s}, t\right)= & A_{0} \exp (-\Gamma t) \cos \left(\mathbf{p} \cdot \mathbf{r}_{s}-\Omega t+\delta\right) \\
= & \frac{1}{2} A_{0} \exp (-\Gamma t+i \delta) \exp \left(i \mathbf{p} \cdot \mathbf{r}_{s}-i \Omega t\right) \\
& +\frac{1}{2} A_{0} \exp (-\Gamma t-i \delta) \exp \left(-i \mathbf{p} \cdot \mathbf{r}_{s}+i \Omega t\right),
\end{aligned}
$$

where $\Gamma$ describes the slow damping of the wave. When Eq. (11) is substituted into Eq. (9), the first term of the Taylor series expansion of the $\exp \left(-2 i k z_{s}\right.$ $\cos \alpha$ ) factor is physically interpreted as reflection of the incident beam by an effective flat mirror, and the second term is interpreted as modulation of the incident beam by the two capillary-wave spatial frequencies $\pm \mathbf{p}$. The modulation of the beam at the liquid surface by a small-amplitude capillary wave is identical to the modulation of the beam at the phase grating by the grating spatial frequencies $\pm \mathbf{K}$.

Last, the light scattered by the liquid surface freely propagates a distance $d_{0}$ to a large-aperture collection lens L 0 of focal length $f_{0}$ and is then focused onto the $x_{o} y_{o}$ output detector plane.

For the geometry of Fig. 1(a), because the electric field of the beam at the exit plane of the phase grating is $E\left(\mathbf{r}_{i}\right)$ $T\left(\mathbf{r}_{i}\right)$, reflection by the effective flat-mirror term of Eq. (9) and then focusing by the collection lens L0 produces the Fourier transform of $E\left(\mathbf{r}_{i}\right) T\left(\mathbf{r}_{i}\right)$ at the detector plane, which is a convolution of the Fourier transform of the incident Gaussian beam with the Fourier transform $\delta$ functions of the three spatial frequencies of $T\left(\mathbf{r}_{i}\right)$. This results in a set of three spots on the detector plane, each having a Gaussian amplitude profile. The central spot is produced by the specularly reflected beam, and the spots to either side of it are produced by the phasegrating first-order diffracted beams. In addition, the 
central beam is modulated at the liquid surface by the two capillary-wave spatial frequencies of Eq. (11) and is also focused onto the detector plane by the collection lens. The Fourier transform of this modulated beam is again the Fourier transform of the central Gaussian beam convolved with the Fourier-transform $\delta$ functions of the capillary-wave spatial frequencies. This produces two additional spots on the detector plane, each having a Gaussian amplitude profile and representing scattering of the incident laser beam by the surface capillary wave. Scattering of the phase-grating first-order diffracted beams by the capillary wave is much weaker and is ignored for the remainder of this paper.

The electric field at the detector plane is then

$$
\begin{aligned}
E_{\mathrm{det}}\left(x_{o}, y_{o}, t\right)= & \exp \left\{i k r_{o}^{2}\left[1-\left(d_{i}+d_{o}\right) / f_{0}\right] / 2 f_{0}\right\} E_{\mathrm{inc}} \\
& \times\left\{\exp \left(-r_{o}^{2} / w_{o}^{2}+i Q_{o} r_{o}^{2}-i \omega t\right)\right. \\
& +\frac{i \epsilon}{2} \exp \left[-\left|\mathbf{r}_{o}-f_{0} \lambda \hat{\eta}^{*} / l\right|^{2}\right. \\
& \left.\times\left(\frac{1}{w_{o}^{2}}-i Q_{o}\right)-i \omega t\right]+\frac{i \epsilon}{2} \\
& \times \exp \left[-\left|\mathbf{r}_{o}+f_{0} \lambda \hat{\eta}^{*} / l\right|^{2}\right. \\
& \left.\times\left(\frac{1}{w_{o}^{2}}-i Q_{o}\right)-i \omega t\right]-i k A_{0} \\
& \times \exp (-\Gamma t+i \delta) \exp \left[-\left|\mathbf{r}_{o}-f_{0} \lambda \hat{\zeta} / \Lambda\right|^{2}\right. \\
& \left.\times\left(\frac{1}{w_{o}^{2}}-i Q_{o}\right)-i(\omega+\Omega) t\right]-i k A_{0} \\
& \times \exp (-\Gamma t-i \delta) \exp \left[-\left|\mathbf{r}_{o}+f_{0} \lambda \hat{\zeta} / \Lambda\right|^{2}\right. \\
& \left.\left.\times\left(\frac{1}{w_{o}^{2}}-i Q_{o}\right)-i(\omega-\Omega) t\right]\right\}, \quad(12
\end{aligned}
$$

where

$$
\begin{aligned}
\hat{\eta}^{*} & =\cos \eta \hat{u}_{x}-\sin \eta \hat{u}_{y}, \\
\hat{\zeta} & =\left(\frac{\cos \xi}{\cos \alpha} \hat{u}_{x}+\sin \xi \hat{u}_{y}\right)\left(\frac{\cos ^{2} \xi}{\cos ^{2} \alpha}+\sin ^{2} \xi\right)^{-1 / 2},
\end{aligned}
$$

(the asterisk denotes that the $y$ coordinate is reversed after reflection from the surface), and where $w_{o}$ and $Q_{o}$ are given by

$$
\begin{aligned}
& w_{o}=\frac{2 f_{0}}{k w_{i}}\left(1+Q_{i}{ }^{2} w_{i}^{4}\right)^{1 / 2}, \\
& Q_{o}=-Q_{i}\left(\frac{w_{i}}{w_{o}}\right)^{2}
\end{aligned}
$$

respectively. The first term in Eq. (12) is the specularly reflected laser beam spot that has the angular frequency $\omega$. The second and the third terms are the phase-grating first-order diffraction spots, which also have frequency $\omega$, that act as reference beams for the heterodyne mixing. The fourth and the fifth terms are the capillary-wave scattered spots whose frequency is Doppler shifted to $\omega \pm \Omega$ by the motion of the capillary wave. The phase-grating first-order diffraction spots and the capillary-wave scattered spots completely spatially overlap at the detector if the propagation direction and the wavelength of the capillary wave satisfy 5

$$
\begin{aligned}
\tan \xi & =\frac{-\tan \eta}{\cos \alpha}, \\
\Lambda & =l\left(\cos ^{2} \eta \cos ^{2} \alpha+\sin ^{2} \eta\right)^{-1 / 2} .
\end{aligned}
$$

In actuality, however, a whole spectrum of capillary waves is continually being thermally created on the liquid surface. Thus a cone of scattered light corresponding to capillary waves with different wavelengths and propagation directions surrounds the specularly reflected central beam. In this case a phase-grating first-order diffraction spot partially overlaps many different capillary-wave scattered spots, producing instrumental broadening. A detector placed at the overlapping spots records the timevarying intensity integrated over the detector face:

$$
I(t)=\int_{\operatorname{det}} \mathrm{d}^{2} r_{0} E_{\operatorname{det}} *\left(\mathbf{r}_{0}, t\right) E_{\mathrm{det}}\left(\mathbf{r}_{0}, t\right) .
$$

The viscoelastic properties of the surface are obtained by analysis of the intensity autocorrelation function,

$$
R(\tau)=\int_{-\infty}^{\infty} \mathrm{d} t I(t+\tau) I(t),
$$

which is theoretically modeled by the ensemble average of the integrand $\langle I(\tau) I(0)\rangle$. This analysis and corrections for instrumental broadening are treated extensively in the literature..$^{2,5,7,9}$

The optical analysis of the surface light scattering geometry of Fig. 1(b) proceeds identically. Lenses L1 and L2 image the phase-grating exit-plane electric field onto the entrance plane of the liquid surface. The electric field at the exit plane of the liquid surface, modulated by the capillary-wave spatial frequencies, is then

$$
\begin{aligned}
E_{\text {surface }}\left(\mathbf{r}_{s}, t\right)= & \exp \left(-r_{i}{ }^{2} f_{1}{ }^{2} / w_{g}{ }^{2} f_{2}{ }^{2}+i Q_{g} f_{1}{ }^{2} r_{i}{ }^{2} / f_{2}{ }^{2}\right) E_{\text {inc }} \\
& \times\left[1+\frac{i \epsilon}{2} \exp \left(i \mathbf{K} \cdot f_{1} \mathbf{r}_{i} / f_{2}\right)\right. \\
& \left.+\frac{i \epsilon}{2} \exp \left(-i \mathbf{K} \cdot f_{1} \mathbf{r}_{i} / f_{2}\right)\right] \\
& \times \exp (-i \omega t) \times\left[1-i k A_{0}\right. \\
& \times \exp (-\Gamma t+i \delta) \exp \left(i \mathbf{p} \cdot \mathbf{r}_{s}-i \Omega t\right) \\
& -i k A_{0} \exp (-\Gamma t-i \delta) \\
& \left.\times \exp \left(-i \mathbf{p} \cdot \mathbf{r}_{s}+i \Omega t\right)\right]
\end{aligned}
$$


where the relation between $\mathbf{r}_{i}$ and $\mathbf{r}_{s}$ at the surface is given by Eq. (5). With second-order terms neglected, this electric field again consists of five spatial frequencies, namely the dc spatial frequency of the specularly reflected beam, the magnified spatial frequencies $\pm f_{1} \mathbf{K} / f_{2}$ of the phase grating, and the spatial frequencies $\pm \mathbf{p}$ of the capillary wave, each modulating the Gaussian amplitude profile of the magnified laser beam. Accordingly, when focused onto the detector plane, the Fourier transform of Eq. (20) is again given by Eq. (12), but with a different overall phase factor. The electric-field 1/e halfwidth of each of the Gaussian spots on the detector plane is

$$
w_{o}=\frac{2 f_{0} f_{1}}{k w_{g} f_{2}}\left\{1+w_{g}{ }^{4}\left[Q_{g}+\frac{k}{2 f_{1}^{2}}\left(f_{1}+f_{2}-d_{i}\right)\right]^{2}\right\}^{1 / 2},
$$

and the wave-front curvature is described by

$$
Q_{o}=-\left[Q_{g}+\frac{k}{2 f_{1}^{2}}\left(f_{1}+f_{2}-d_{i}\right)^{2}\right]\left(\frac{w_{g}}{w_{o}}\right)^{2} .
$$

In the analysis of this section, the slow evolution of the transverse beam profile $F$ as a function of $z_{i}$ along the liquid surface was ignored. In this case the effective flat-mirror term of Eq. (9) served only to fold the optical path. The distortion introduced into the profile of the reflected beam caused by the weak $z_{i}$ dependence of $F$ along the liquid surface is examined in Appendix A.

\section{Optical Analysis of the Hybrid Reflection-Transmission Surface Light-Scattering Geometry}

A hybrid reflection-transmission instrument for surface light scattering that completely compensates for surface sloshing is shown in Fig. 3(a). The incident laser beam propagates along the symmetry axis of spherical mirror M1 of radius $R$. A transparent dish is filled with the liquid to be studied to a depth $D \ll$ $R$ and is centered over the mirror. The liquid surface is the height $R$ above the mirror and the beam is normally incident upon the liquid. The electric field at the exit plane of the grating is imaged onto the liquid surface by the two lenses L1 and L2. Light is transmitted through the liquid (i.e., the first pass), reflects off the spherical mirror, and retraces its original path through the liquid (i.e., the second pass). The beam plus scattered light is then reflected by half-silvered mirror M2 and is focused onto the detector plane by collection lens L0. Light is scattered by the surface capillary waves in this geometry three times, reflection scattering at the first pass, transmission scattering at the first pass, and transmission scattering at the second pass. If the liquid surface sloshes, as in Fig. 3(b), the first-pass reflection scattered light is strongly deflected. But the light transmitted at the first pass is reflected by the spherical mirror, retraces its original path through the liquid,
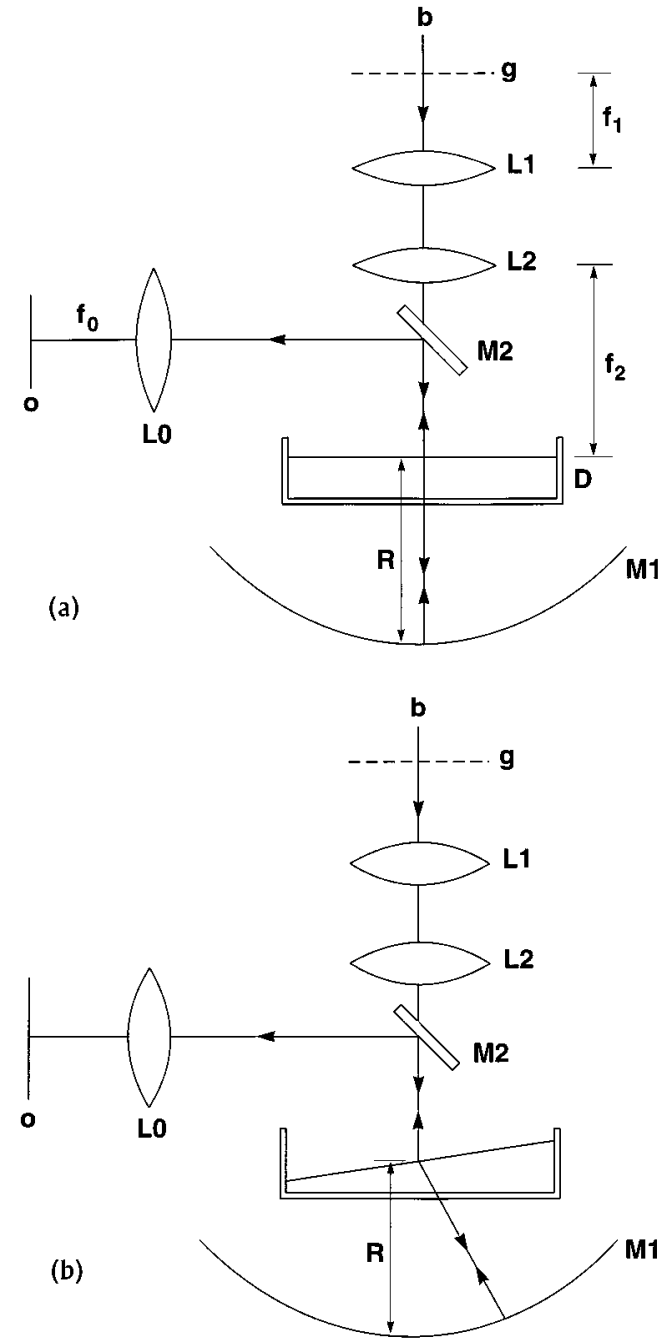

Fig. 3. (a) Hybrid reflection-transmission surface light-scattering design that completely compensates for surface sloshing. Gaussian beam $b$ is transmitted through phase grating $g$ and is imaged onto the liquid surface by lenses L1 and L2. The transmitted beam is reflected by spherical mirror $\mathrm{M} 1$ of radius $R$, passes through the liquid a second time, is reflected by the half-silvered mirror M2 and is focused onto the detector plane o by lens L0. The depth of the liquid is $D \ll R$. (b) When the liquid sloshes, the transmitted beam is deflected but retraces its original path after being reflected by M1 because the liquid surface is the distance $R$ above the spherical mirror.

and is focused to the same place on the detector plane as before.

This geometry, however, has the following drawback. Because the focal length of the spherical mirror is

$$
f_{3}=R / 2,
$$

the electric field at the exit plane of the first-pass liquid surface, which is modulated by the spatial frequencies of the surface capillary waves, is imaged with negative unit magnification onto the entrance plane of the second-pass surface. Thus each capillary wave appears twice at the second-pass surface; 


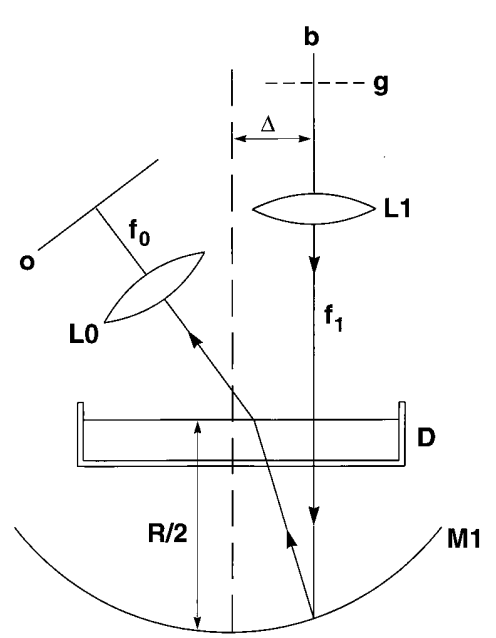

Fig. 4. Gaussian beam b is offset a distance $\Delta$ from the axis of spherical mirror M1. The beam is transmitted through phase grating $g$ and is focused onto the liquid surface by lens L1. The transmitted beam is reflected by spherical mirror M1 of radius $R$, passes through the liquid a second time, and is focused onto detector plane o by lens L0. The depth of the liquid is $D \ll R$, and the distance between the liquid surface and the spherical mirror is $R / 2$.

the wave itself propagates in the $\xi$ direction and its image propagates in the $-\xi$ direction. As a result, the two spatially overlapping sets of capillary-wave scattered spots on the detector plane coherently interfere, and the two Doppler-shifted frequencies $\omega \pm$ $\Omega$ at each coherent overlap spot produce a complicated heterodyne signal when superposed with a phase-grating first-order diffraction spot.

A variant of this geometry that spatially separates the first-pass and the second-pass capillary-wave scattered spots on the detector plane is shown in Fig. 4. Lens L1 focuses the phase-grating exit-plane electric field onto the first-pass liquid surface. Because the liquid surface is now the distance $R / 2$ above the spherical mirror, the combination of spherical mirror M1 plus final collection lens L0 images the exit plane of the first-pass surface onto the detector plane. The central specularly reflected Gaussian profile spot on the detector plane is modulated by the first-pass capillary-wave spatial frequencies. The second-pass capillary-wave spatial frequencies are Fourier transformed onto the detector plane by collection lens L0 and are thus spatially separated from the specularly reflected spot. The second-pass spots may then be made to overlap the spots produced by the phase-grating first-order diffracted beams that were focused onto the first-pass liquid surface and then imaged from there onto the detector plane. The modulation of the phase-grating spots by the first-pass capillary waves is a small effect and is ignored here. The incoming laser beam is purposely offset a distance $\Delta \ll R$ from the spherical-mirror symmetry axis so as to steer the second-pass transmitted central beam and its surrounding cone of capillary-wave scattered light away from the firstpass reflected central beam and its surrounding cone of capillary-wave scattered light. Experimentally, offsetting the incident beam in the plane of the grating beams or in the plane perpendicular to the grating beams gave identical results.

For the geometry of Fig. 4, the grating spots are located at

$$
\mathbf{r}_{o}=\frac{2 f_{1} f_{0} \lambda}{R l} \hat{\eta}^{*}
$$

the second-pass scattered spots are located at

$$
\mathbf{r}_{o}=\frac{\lambda f_{0}}{\Lambda} \hat{\xi}\left[1+O\left(\Delta^{2} / R^{2}\right)\right]
$$

$\hat{\xi}=\hat{\zeta}$ at normal incidence, and the Gaussian amplitude profile of each of the spots has the electric-field $1 / e$ half-width

$$
w_{o}=\frac{2 f_{1} f_{0} \lambda}{\pi R w_{g}} .
$$

The correction to Eq. (25) due to the incident beam's being off axis in Fig. 4 is of the order of $\Delta^{2} / R^{2}$ and is ignored here. Perfect spatial overlap of the secondpass scattered spots and the phase-grating first-order diffraction spots occurs when the propagation direction and the amplitude of the capillary wave satisfy

$$
\begin{gathered}
\xi=-\eta \\
\Lambda=\frac{l R}{2 f_{1}} .
\end{gathered}
$$

Last, we consider the surface of the liquid as a whole tilting by the small angle $\phi$ because of sloshing in response to low-frequency external vibrations. Carrying out a ray-tracing calculation of the central transmitted beam and the phase-grating diffracted beams, we find that, to first order, they deflect through the angle

$$
\phi_{\text {deflected }}=2(n-1) \phi\left[\frac{A}{R}+\frac{(n-1) D}{n R}\right],
$$

where $A$ is the equilibrium distance between the center of curvature of spherical mirror M1 and the liquid surface and $D$ is the equilibrium depth of the liquid in the transparent dish. In order to image the exit plane of the first-pass liquid surface onto the detector plane, we require that

$$
B+n D=(R / 2),
$$

where $B$ is the distance between the bottom of the dish and the spherical mirror surface and

$$
A+B+D=R .
$$

Combining Eqs. (28)-(30), we obtain

$$
\phi_{\text {deflected }}=(n-1) \phi\left[1+\frac{2\left(n^{2}-1\right) D}{n R}\right] \text {, }
$$




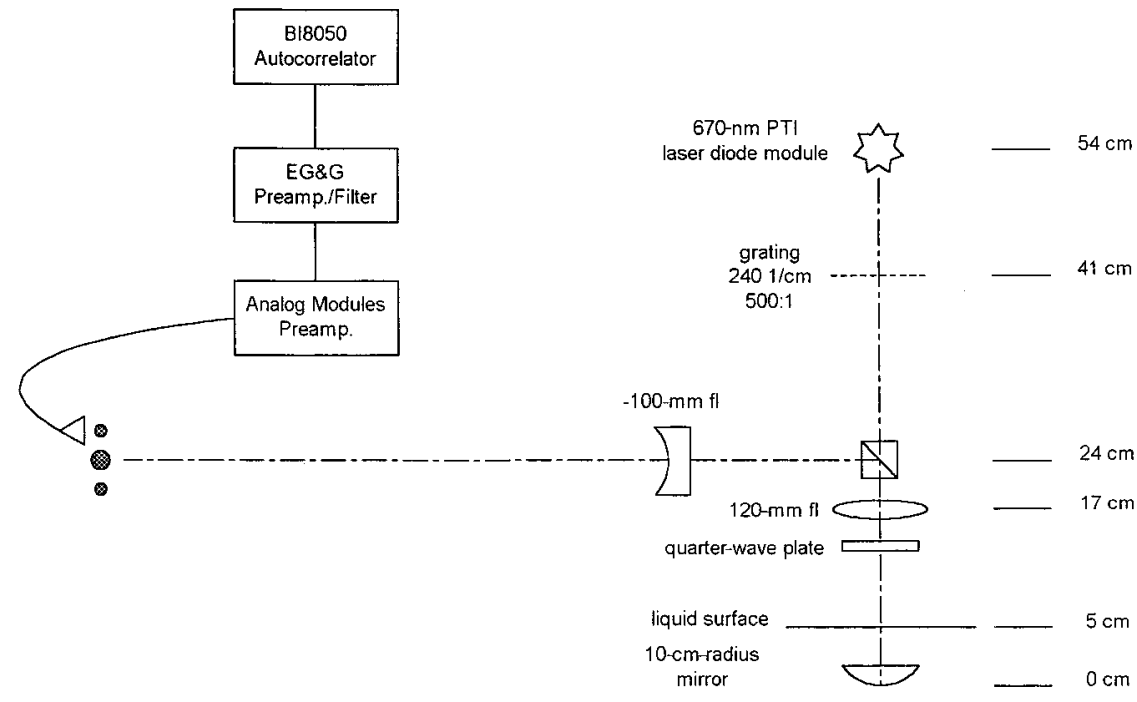

Fig. 5. Optical arrangement for the transmission configuration used to produce the data in Table 1. fl, focal length. which for $D \ll R$ is comparable with the decreased sensitivity to surface sloshing encountered in the single-pass transmission geometry of Ref. 10. As the surface sloshes, the leading corrections to Eq. (25) for the positions of second-pass scattered spots on the detector plane are of the order of $(\Delta / R) \phi$ and $\phi^{2}$ and are ignored here.

\section{Experiment}

The experimental arrangement depicted in Fig. 4 was implemented as shown in Fig. 5. A PTI 670-nmwavelength laser diode module was used as the source of illumination. A custom-made phase grating was designed with $K=240 \mathrm{~cm}^{-1}$ and a 500:1 contrast ratio and was optimized to produce predominantly zero- and first-order diffraction spots. Spindler-Hoyer Microbench components were used to assemble the instrument on a Newport Research optical table. The various distances along the optical path given in Fig. 5 were known to within a few millimeters. A diode detector with a $1-\mathrm{mm}^{2}$ active area was positioned to receive an entire first-order diffraction grating beam in the detector plane. The grating beam provided the reference beam for heterodyne mixing with the surface scattered light and was sufficiently intense to drive the detector well above the noise level without saturating the detector circuit. A preamplifier was attached directly to the photodiode, and the output was ac coupled to a lownoise bandpass amplifier. The high-pass roll-off was set to $\sim 1-2 \mathrm{kHz}$ and the low pass was set to $100 \mathrm{kHz}$ or higher. The instrument was comparatively insensitive to environmental noise. It is the practice in the field to isolate the cell carefully from both building vibrations that couple through the table and acoustic noise. But with this configuration, one could tap the tabletop and observe only a minor amount of beam jitter on the face of the photodetector.

The conditioned signal was analyzed by a Brookhaven Instruments BI-8050 autocorrelator with analog input. The output files were fit by use of a model autocorrelation function of the form

$$
R(\tau)=A_{0} \exp (-\Gamma \tau) \cos (\Omega \tau)+C
$$

and were used to compute the surface tension by use of the dispersion equation of Refs. 5 and 15, which for pure liquids is equivalent to the Levich equation ${ }^{1}$ when the small contribution from the vapor phase is ignored. Our dispersion equation included the vapor-phase contribution.

Data were collected on acetone, ethanol, and water. The acetone and ethanol were of spectroscopic grade, and the water was double distilled and passed the shake test for residual surfactant. A representative autocorrelation function for acetone is shown in Fig. 6. The Fourier transform of a representative autocorrelation function for ethanol is shown in Fig. 7. The water data were of the same quality. The bottom curve in each of these figures is a plot of the residuals for each fit with Eq. (32) or its Fourier

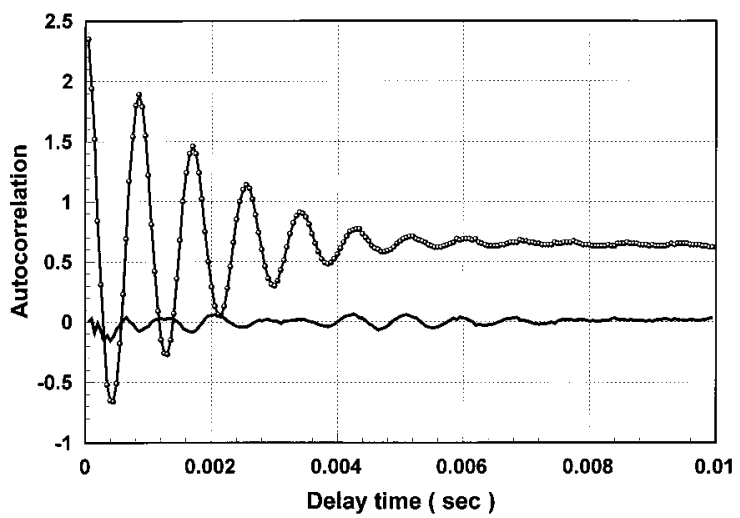

Fig. 6. Typical correlogram for acetone at room temperature $\left(\sim 22^{\circ} \mathrm{C}\right)$. The optical table was not elevated so that mechanical vibration was allowed to couple to the cell that in this case was open at the top and had an optical-quality bottom window. The residuals show more structure than is usual. 
Table 1. Comparison of Backcomputed Wave-Vector Values for Acetone, Ethanol, and Water ${ }^{a}$

\begin{tabular}{|c|c|c|c|c|c|c|}
\hline & \multicolumn{2}{|c|}{ Acetone } & \multicolumn{2}{|c|}{ Ethanol } & \multicolumn{2}{|c|}{ Water } \\
\hline & $\begin{array}{c}\text { Frequency } \\
(\mathrm{kHz})\end{array}$ & $\begin{array}{c}q \text {-calc } \\
(1 / \mathrm{cm})\end{array}$ & $\begin{array}{c}\text { Frequency } \\
(\mathrm{kHz})\end{array}$ & $\begin{array}{c}q \text {-calc } \\
(1 / \mathrm{cm})\end{array}$ & $\begin{array}{c}\text { Frequency } \\
(\mathrm{kHz})\end{array}$ & $\begin{array}{l}q \text {-calc } \\
(1 / \mathrm{cm})\end{array}$ \\
\hline & 9.752 & 504.3 & 9.233 & 488.5 & 15.04 & 498.3 \\
\hline & 9.734 & 503.7 & 9.228 & 488.3 & 15.05 & 498.5 \\
\hline & 9.748 & 504.2 & 9.218 & 488.0 & 15.05 & 498.5 \\
\hline Averages & 9.745 & 504.0 & 9.226 & 488.3 & 15.05 & 498.4 \\
\hline
\end{tabular}

${ }^{a}$ Overall average 496.9 , overall standard deviation 6.9 .

transform. In each case the center capillary-wave frequency $\Omega$ and the damping time $\Gamma$ were estimated to a precision of $10 \mathrm{kHz}$ or better by use of a weighted, nonlinear, least-squares-fitting procedure. The estimate of the cross correlation of the parameters was nearly zero. The residuals showed some structure that comes in part from the instrument response function that was not included in these fits. Rather, the procedure of Ref. 5 was used to correct $\Omega$ and $\Gamma$. The correction turned out to be small for $\Omega$ but was substantial for $\Gamma$. The results for acetone, ethanol, and water were used with the known values for the surface tension and density to backcalculate the value of the wave number of the surface capillary wave that was measured by the system. The results of the calculation are shown in Table 1; the obtained wave numbers are consistent to within $\pm 1.4 \%$, and the variability of the results for a given fluid corresponds to an error in the inferred surface tension of $\pm 4 \%$. When a traditional reflection system that is well isolated from external vibrations is used, the surface tension may be obtained to within a few tenths of a percent. These preliminary data provide evidence that the apparatus was working properly and supports our conclusion that the instrument is capable of accurately determining the surface lightscattering autocorrelation function. The next step in the development in the instrument is to optimize the experimental parameters and perform a more

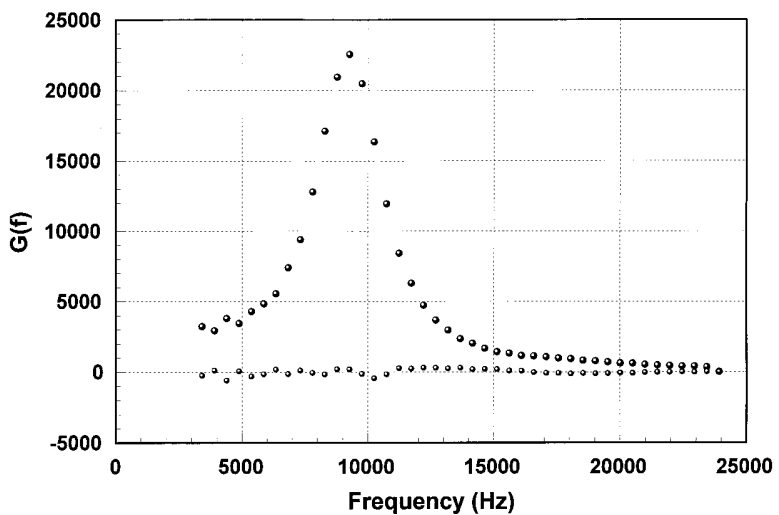

Fig. 7. Fourier transform of a typical correlogram obtained for ethanol under the same conditions as Fig. 6. The residual of the nonlinear least-squares fit to the spectrum of Eq. (32) is shown. The few data points below $2 \mathrm{kHz}$ showed the distortion due to the high-pass filter and were excluded from the fit. careful data analysis that includes the instrument function.

A reflection cat's eye instrument was also constructed in which the optical axis of the input beam was tilted off normal and the spherical mirror was placed to catch the reflected beam. Gallium was used as the reflecting liquid just above its melting point $\left(29.78^{\circ} \mathrm{C}\right)$. Without the spherical mirror, the system was prone to slosh because of building vibrations and acoustical noise. We demonstrated that a clean correlogram could be obtained with the cat's eye reflection system even under such conditions. Detailed results will be presented elsewhere.

\section{Discussion}

The theory developed here is in the spirit of Ref. 9 but has been done in greater detail and has been applied to a new optical arrangement. We have shown details of the propagation of a Gaussian beam with spherical phase fronts for the reflection system of the original design of Hard et al., ${ }^{2}$ the reflection imaging system of Ref. 9, and the transmission cat's eye geometry. We show how the intensity is distributed in the detector plane as a result of the superposition of the diffracted field from the phase grating placed before the surface and the field scattered by the liquid surface covered by a spectrum of capillary waves.

The liquid surface profile is generated by abrupt, random pressure fluctuations coupled to the surface, forming a random field of capillary waves that distribute over wavelengths from nanometers to millimeters. Scattered beams within only a narrow range of wave numbers that spatially overlap the reference beam, however, contribute to the intensity fluctuations recorded by the photodetector. Naturally the wave-number filter provided by the optical system is not perfect because the beams are Gaussian, so that spots on the detector surface are not infinitesmally small and thus partial overlapping of the grating spots with the scattered spots occurs. This imperfection must be taken into account in a complete analysis and, indeed, the exact correction formulas are known, ${ }^{5,9}$ assuming that the paraxial approximation required for the validity of Fourier optics is satisfied.

The cat's eye optical system with such a refined data-analysis capability will provide a compact instrument, relatively insensitive to environmental noise, that will yield detailed information about in- 
terfacial dynamics beyond the determination of merely the interfacial tension. The transmission system examined here is intrinsically able to handle low levels of slosh, while the traditional reflection system is sensitive to low-frequency ripples pumped up by any environmental noise. Our experience is that, once aligned, the cat's eye instrument can be used on a common laboratory bench by technicians with minimal training in light-scattering spectroscopy.

\section{Appendix A}

If a beam is incident upon a flat mirror and the evolution of its transverse profile along the mirror surface is ignored, reflection by the flat mirror does not distort the beam profile. It serves only to fold the optical path. In this appendix we calculate the distortion produced by reflection from a flat surface in the path of a Gaussian beam that has been previously modulated by a phase grating as in Figs. 1(a) and 1(b). It is our belief that this correction has not been examined previously.

The amplitude profile of the central beam and the two first-order diffraction grating beams of Fig. 1(a) in the vicinity of the flat reflecting surface is

$$
\begin{aligned}
F\left(x_{i}, y_{i}, z_{i}\right) \approx & \exp \left(i k r_{i}{ }^{2} / 2 d_{i}\right)\left\{\exp \left[-r_{i}{ }^{2}\left(\frac{1}{w_{s}{ }^{2}}+i Q_{s}\right)\right]\right. \\
& +\frac{i \epsilon}{2} \exp \left[-\left|\mathbf{r}_{i}-\frac{d_{i}+z_{i}}{k} \mathbf{K}\right|^{2}\left(\frac{1}{w_{s}{ }^{2}}+i Q_{s}\right)\right] \\
& +\frac{i \epsilon}{2} \exp \left[-\left|\mathbf{r}_{i}+\frac{d_{i}+z_{i}}{k} \mathbf{K}\right|^{2}\right. \\
& \left.\left.\times\left(\frac{1}{w_{s}^{2}}+i Q_{s}\right)\right]\right\},
\end{aligned}
$$

$$
w_{s} \approx \frac{2 d_{i}}{k w_{g}}\left[1+\left(Q_{g}+\frac{k}{2 d_{i}}\right)^{2} w_{g}^{4}\right]^{1 / 2}
$$

is the electric-field $1 / e$ half-width of each of the beams and

$$
Q_{s} \approx \frac{-k^{2} w_{g}^{4}}{4 d_{i}^{2}}\left(Q_{g}+\frac{k}{2 d_{i}}\right)^{2}\left[1+\left(Q_{g}+\frac{k}{2 d_{i}}\right)^{2} w_{g}^{4}\right]^{-1}
$$

describes the curvature of the wave fronts. The weak dependence of $w_{s}$ and $Q_{s}$ on $z_{i}$ is ignored in approximations (A2) and (A3) because the effect of $z_{i}$ is much smaller than the dominant correction that we now examine.

The flat reflecting surface cuts the three beams obliquely, as is illustrated in Fig. 8, so that the separation between the central beam and one of the first order diffraction grating beams at the reflecting surface is larger than the separation between the central beam and the other grating beam. When approximation (A1) with $z_{i}=x_{s} \sin \alpha$ is substituted into Eq.

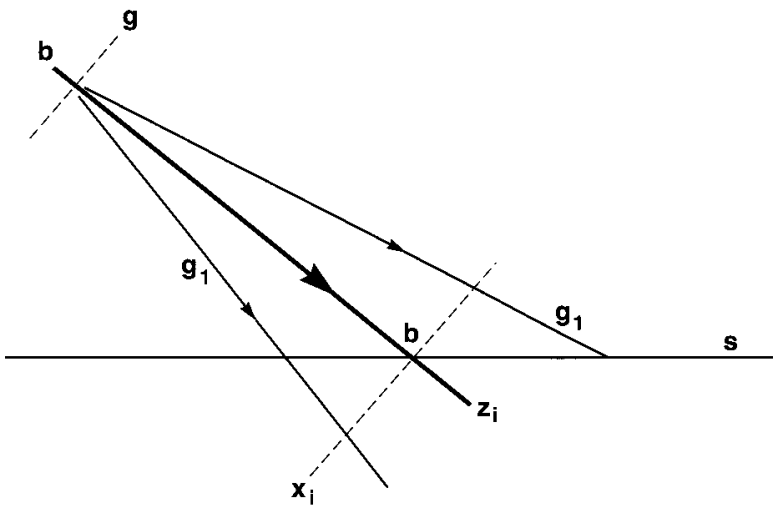

Fig. 8. Beam b is transmitted through phase grating $g$ and is diagonally incident upon flat surface s. The two-phase-grating first-order diffracted beams are denoted by $g_{1}$. The distance on the surface between the central beam and one of the diffracted beams is larger than the distance between the central beam and the other diffracted beam.

(8) and then the focusing of the reflected beams onto the detector plane by collection lens L0 is calculated, the phase-grating diffraction spots on the detector plane are centered at

$$
\begin{aligned}
& x_{o} \approx \pm \frac{f_{0} \lambda}{l} \cos \eta[1 \pm(\lambda / l) \tan \alpha \cos \eta] \\
& y_{o} \approx \mp \frac{f_{0} \lambda}{l} \sin \eta
\end{aligned}
$$

rather than at the result of Eq. (12). The distortion produced by the flat reflecting surface's cutting the three diverging beams obliquely causes the grating to appear to have the periodicity $l^{\prime}$, which is given by

$$
l^{\prime}=l\left[1 \mp(\lambda / l) \tan \alpha \cos ^{3} \eta+O\left(\lambda^{2} / l^{2}\right)\right]
$$

and the angle of orientation $\eta^{\prime}$, which is given by

$$
\tan \eta^{\prime}=-\tan \eta\left[1 \mp(\lambda / l) \tan \alpha \cos \eta+O\left(\lambda^{2} / l^{2}\right)\right] .
$$

Distortion does not occur if the grating rulings are parallel to the $x_{i}$ axis. For $\lambda=0.5145 \mu \mathrm{m}, l=40$ $\mu \mathrm{m}$, and $\alpha=45^{\circ}$, the largest change in the apparent phase-grating periodicity is $\left(l^{\prime}-l\right) / l=1.3 \%$.

For the imaging geometry of Fig. 1(b) the phase grating is in perfect focus on the flat reflecting surface at $x_{s}=0$ and is slightly misfocused at $x_{s} \neq 0$, as illustrated in Fig. 9. Because it is relatively easy to calculate the consequences of misfocus for a single spatial frequency $q$, we express the Gaussian modu- 


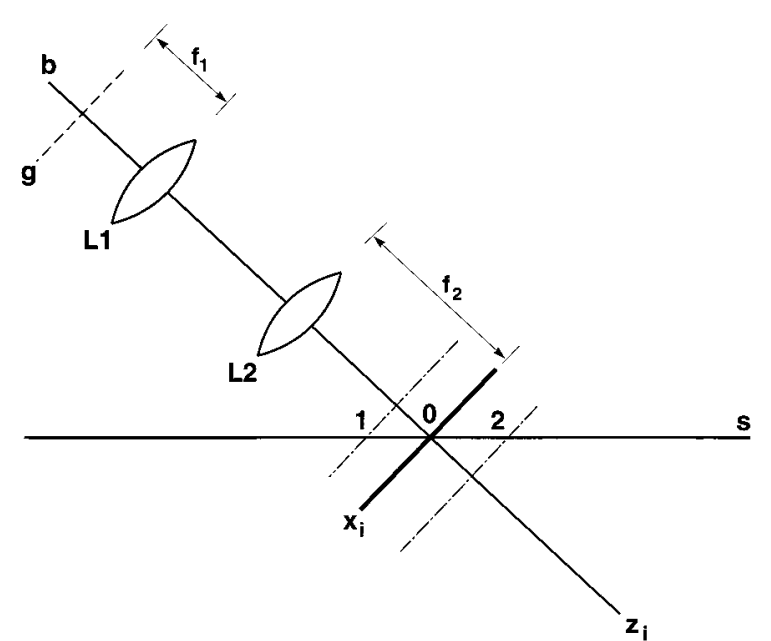

Fig. 9. Beam $b$ is transmitted through phase grating $g$ and is imaged by lenses L1 and L2. The image is in perfect focus at the location 0 on flat surface $\mathrm{s}$ (solid line). The image at locations 1 and 2 on the surface is slightly misfocused (dotted-dashed lines).

lated beam profile in the vicinity of the flat reflecting surface as the spectrum of spatial frequencies:

$$
\begin{aligned}
F\left(x_{i} y_{i} z_{i}\right) \approx & \int \mathrm{d}^{2} q \exp \left(-i q^{2} f_{1}^{2} z_{i} / 2 k f_{2}^{2}\right) \\
& \times\left[A_{0}(\mathbf{q})+\frac{i \epsilon}{2} A_{+}(\mathbf{q}) \exp \left(-i f_{1} \mathbf{q} \cdot \mathbf{r}_{i} / f_{2}\right)\right. \\
& \left.+\frac{i \epsilon}{2} A_{-}(\mathbf{q}) \exp \left(i f_{1} \mathbf{q} \cdot \mathbf{r}_{i} / f_{2}\right)\right]
\end{aligned}
$$

where

$$
\begin{aligned}
& A_{0}(\mathbf{q})=\exp \left[\left(-w_{g}{ }^{2} q^{2}\right) / 4\left(1-i Q_{g} w_{g}{ }^{2}\right)\right], \\
& A_{+}(\mathbf{q})=\exp \left[\left(-w_{g}{ }^{2}|\mathbf{q}-\mathbf{K}|^{2}\right) / 4\left(1-i Q_{g} w_{g}{ }^{2}\right)\right], \\
& A_{-}(\mathbf{q})=\exp \left[\left(-w_{g}{ }^{2}|\mathbf{q}+\mathbf{K}|^{2}\right) / 4\left(1-i Q_{g} w_{g}{ }^{2}\right)\right] .
\end{aligned}
$$

Again, when Eqs. (A8) with $z_{i}=x_{s} \sin \alpha$ are substituted into Eq. (8) and then the focusing of the reflected beam onto the detector plane by collection lens L0 is calculated, the phase-grating first-order diffraction spots on the detector plane are centered at

$$
\begin{aligned}
& x_{o}= \pm f_{0}\left(\frac{f_{1} \lambda}{f_{2} l}\right)\left(\cos \eta-f_{1} \lambda \tan \alpha / 2 f_{2} l\right), \\
& y_{o}=\mp f_{0}\left(\frac{f_{1} \lambda}{f_{2} l}\right) \sin \eta,
\end{aligned}
$$

rather than at the result of Eq. (12). The distortion produced by the image misfocus causes the grating to appear to have the periodicity $l^{\prime}$ given by

$$
l^{\prime}=\frac{f_{2} l}{f_{1}}\left[1 \pm f_{1} \lambda \tan \alpha \cos \eta / 2 f_{2} l+O\left(\lambda^{2} / l^{2}\right)\right]
$$

and the angle of orientation $\eta^{\prime}$ given by

$$
\tan \eta^{\prime}=\frac{-\sin \eta}{\cos \eta-f_{1} \lambda \tan \alpha / 2 f_{2} l}
$$

Again no distortion occurs for $\eta=90^{\circ}$. For $f_{1}=f_{2}$ and the parameters of the previous example, the largest change in the apparent phase-grating periodicity is $\left(l^{\prime}-l\right) / l=0.6 \%$. For a phase grating with $K=$ $500 \mathrm{~cm}^{-1}$, this represents a $3-\mathrm{cm}^{-1}$ error, which is considered to be large.

We thank Lars Lading for many conversations about the instrumentation of surface light-scattering spectroscopy. We also thank Code UG (Microgravity Science and Application Division) at NASA Headquarters for support.

\section{References}

1. D. Langevin, Light Scattering by Liquid Interfaces and Complementation Techniques, Vol. 41 of Surfactant Science Series (Dekker, New York, 1992).

2. S. Hard, Y. Hamnerius, and O. Nilsson, "Laser heterodyne apparatus for measurements of liquid surface propertiestheory and experiments," J. Appl. Phys. 47, 2433-2442 (1976).

3. R. H. Katyl and U. Ingard, "Line broadening of light scattered from a liquid surface," Phys. Rev. Lett. 19, 64-66 (1967).

4. R. H. Katyl and U. Ingard, "Scattering of light by thermal ripplons," Phys. Rev. Lett. 20, 248-249 (1968).

5. R. V. Edwards, R. S. Sirohi, J. A. Mann, L. B. Shih, and L. Lading, "Surface fluctuation scattering using grating heterodyne spectroscopy," Appl. Opt. 21, 3555-3568 (1982).

6. L. B. Shih, "Surface fluctuation spectroscopy: a novel technique for characterizing liquid interfaces," Rev. Sci. Instrum. 55, 716-726 (1984).

7. J. A. Mann and R. V. Edwards, "Surface fluctuation spectroscopy: comments on experimental technique and capillary ripple theory," Rev. Sci. Instrum. 55, 727-731 (1984).

8. E. Mazur and D. S. Chung, "Light scattering from the liquidvapor interface," Physica 147A, 387-406 (1987).

9. L. Lading, J. A. Mann, and R. V. Edwards, "Analysis of a surface scattering spectrometer,” J. Opt. Soc. Am. A 6, 16921701 (1989).

10. K. Sakai, P.-K. Choi, H. Tanaka, and K. Takagi, "A new light scattering technique for wideband ripplon spectroscopy at the MHz region,” Rev. Sci. Instrum. 62, 1192-1195 (1991).

11. T. M. Jorgensen, "A low-cost surface light scattering spectrometer," Meas. Sci. Technol. 3, 588-595 (1992).

12. W. M. Klipstein, J. S. Radnich, and S. K. Lamoreaux, "Thermally excited liquid surface waves and their study through the quasielastic scattering of light," Am. J. Phys. 64, 758-765 (1996).

13. L. Lading, Department of Information Technology, Riso National Laboratory, DK-4000 Roskilde, Denmark (personal communication, September 1992).

14. J. W. Goodman, Introduction to Fourier Optics (McGraw-Hill, New York, 1968), Chap. 5.

15. J. A. Mann, "Dynamics, structure, and function of interfacial regions," Langmuir 1, 10-23 (1985). Also see J. A. Mann, "Dynamic surface tension and capillary waves," Surf. Colloid Sci. 13, 145-212 (1984). The original construction of the dispersion equation was in J. A. Mann, J. F. Baret, F. J. Dechow, and R. S. Hansen, "Interfacial forces and the Brillouin spectrum of the interfacial light scattering power," J. Colloid Interface. Sci. 37, 14-32 (1971). 